\title{
Vibrational Spectroscopy of Binary Titanium Borides: First-Principles and Experimental Studies
}

\author{
Urszula D. Wdowik, ${ }^{1}$ Agnieszka Twardowska, ${ }^{1}$ and Bogusław Rajchel ${ }^{2}$ \\ ${ }^{1}$ Institute of Technology, Pedagogical University, Podchorazych 2, 30-084 Krakow, Poland \\ ${ }^{2}$ Institute of Nuclear Physics, Polish Academy of Sciences, Radzikowskiego 152, 31-342 Krakow, Poland \\ Correspondence should be addressed to Urszula D. Wdowik; sfwdowik@cyf-kr.edu.pl
}

Received 30 August 2016; Accepted 1 December 2016; Published 3 January 2017

Academic Editor: Da-Ren Hang

Copyright (C) 2017 Urszula D. Wdowik et al. This is an open access article distributed under the Creative Commons Attribution License, which permits unrestricted use, distribution, and reproduction in any medium, provided the original work is properly cited.

\begin{abstract}
Vibrational dynamics of binary titanium borides is studied from first-principles. Polarized and unpolarized Raman spectra of TiB, $\mathrm{TiB}_{2}$, and $\mathrm{Ti}_{3} \mathrm{~B}_{4}$ are reported along with the experimental spectra of commercial powder and bulk $\mathrm{TiB}_{2}$ containing less than $1 \mathrm{wt} . \%$ of impurity phases. The X-ray diffraction spectroscopy, applied for phase composition examination of both bulk and powder materials, identifies only the $\mathrm{TiB}_{2}$ phase. The simulated Raman spectra together with literature data support interpretation and refinement of experimental spectra which reveal components arising from titanium dioxide $\left(\mathrm{TiO}_{2}\right)$ and amorphous boron carbide $\left(\mathrm{B}_{4} \mathrm{C}\right)$ impurity phases as well as graphitic carbon. These contaminations are the by-products of synthesis, consolidation, and sintering aids employed to fabricate powder and bulk titanium diboride.
\end{abstract}

\section{Introduction}

Generally, the Ti-B system comprises three compounds, namely, $\mathrm{TiB}, \mathrm{TiB}_{2}$, and $\mathrm{Ti}_{3} \mathrm{~B}_{4}$ [1]. These borides have attracted much experimental and theoretical research because of their unique properties such as high melting point, high hardness, high elastic modulus, good thermal and electrical conductivity, excellent oxidation resistance, and considerable chemical stability [2]. The combination of these properties makes titanium borides promising materials for multifunctional applications, for example, electrode materials, cutting tools, wear-resistant parts, protecting coatings, and all kinds of high-temperature structural components [3].

The most extensively studied $\mathrm{TiB}_{2}$ compound has recently gained renewed experimental interest due to its application for deposition of thin Ti-B films [4-6]. We note that chemical composition of $\mathrm{Ti}-\mathrm{B}$ film varies with applied conditions of deposition, and hence the phase composition of deposited material differs from that desired and expected. An analysis of the phase composition of thin and frequently amorphous (or partially amorphous) Ti-B films by the X-ray diffraction (XRD) method remains uncertain due to the content of light boron element. Therefore, for characterization of deposited thin Ti-B films, some complementary methods, such as the Raman spectroscopy, have to be applied. On the other hand, reference Raman spectra are usually based on measurements carried out on samples prepared in different conditions, and thus the resulting Raman spectra show differences in position, intensities, and even the number of peaks among the spectra recorded for the same phase and analyzed in very similar experimental conditions. In order to resolve these ambiguities one may calculate positions and intensities of the Raman-active modes for a given system using the currently available theoretical tools such as those based on state-of-the-art density functional theory (DFT). Results of numerical simulations can then be used for interpretation and refinement of respective experimental spectra.

So far, a number of theoretical and experimental works have been done to investigate the structural, electronic, and elastic properties of titanium borides [7-11], leaving their dynamical properties highly unexplored [12, 13]. This research extends and supplements the present knowledge on titanium borides by providing information on their vibrational properties. In particular, the positions and intensities of the Raman-active phonons of $\mathrm{TiB}, \mathrm{TiB}_{2}$, and $\mathrm{Ti}_{3} \mathrm{~B}_{4}$ are determined form first-principles calculations by using the DFT 
theory and the direct method. We also provide interpretation of the Raman spectra measured for commercially available powder and bulk samples of titanium diboride. Results of these studies are hoped to stimulate further experimental and theoretical progress in the field of $\mathrm{Ti}-\mathrm{B}$ system.

\section{Methodology}

2.1. Experimental. Experiments were performed for commercial $\mathrm{TiB}_{2}$ powder (H. C. Starck, Germany) and bulk (target, Goodfellow, UK) samples. The grain size of $\mathrm{TiB}_{2}$ powder with purity of about $99 \mathrm{wt} . \%$ was in the range $2.5-3.5 \mu \mathrm{m}$. The target sample of $30 \mathrm{~mm}$ in diameter and $4 \mathrm{~mm}$ in thickness was mechanically polished on one side using diamond grinding $(9,6$, and $3 \mu \mathrm{m})$ and finally polished in $1 \mu \mathrm{m}$ suspension. At each polishing step, the surface was degreased by 2 Propanol and then ultrasonically cleaned in acetone bath for 5 minutes. After drying (in air), the target was mounted in a vacuum chamber to perform ion cleaning at room temperature and pressure of $10^{-2} \mathrm{~Pa}$. The iron cleaning was done by a beam of $\mathrm{Ar}^{+}$ions of energy of $10 \mathrm{keV}$ directed at sample at an angle of $65^{\circ}$ (measured to the normal of target surface). Such preparation procedure is required for the Raman measurements as the spectrometer used in our studies is equipped with the confocal (light) microscope. Moreover, the target is further used for deposition of $\mathrm{TiB}_{2}$ thin films by the PVD method (results not discussed in the present paper).

Phase identification was performed by the X-ray diffraction (XRD) method using PANalytical Empyrean diffractometer. The $\mathrm{CuK}_{\alpha}$ radiation (intended $\lambda=1.5406 \AA$, intensity ratio $\mathrm{CuK}_{\alpha_{1}} / \mathrm{CuK}_{\alpha_{2}}=2, U=40 \mathrm{kV}, I=30 \mathrm{~mA}$ ) in the Bragg-Brentano configuration was used for this purpose. The XRD patterns were collected in $2 \Theta$ geometry over the scattering angles ranging from $20^{\circ}$ to $82^{\circ}$ with a step size of $0.02^{\circ}$. Analysis was performed according to the ICSD database and the Rietveld method which took into account the ratio $\mathrm{CuK}_{\alpha_{1}} / \mathrm{CuK}_{\alpha_{2}}=2$.

The Raman spectroscopy was applied to refine the phase composition of both powder and bulk (target) samples. To excite Raman spectra, the Nd:YAG laser beam with wavelength of $532 \mathrm{~nm}$ and a power of $6.25 \mathrm{~mW}$ was used. Unpolarized Raman spectra in backscattering geometry were collected at room temperature using the Thermo-Nicolet Raman ALMEGA XR dispersive confocal spectrometer operating in the micro-Raman mode. Raman spectra were recorded with normal $\left(4 \mathrm{~cm}^{-1}\right)$ and high-spectral $\left(2 \mathrm{~cm}^{-1}\right)$ resolutions.

2.2. Theoretical. Calculations were carried out within the DFT method implemented in the VASP code $[14,15]$. Electron-ion interaction was represented by the projector augmented wave (PAW) method. The generalized gradient approximation with parametrization of Perdew, Burke, and Ernzerhof (GGA-PBE) [16, 17] was applied for the exchange and correlation potential. The wavefunctions were expanded in a plane-wave basis set with a cutoff energy of $420 \mathrm{eV}$. Reference configurations for valence electrons were $\left(3 \mathrm{~d}^{3} 4 \mathrm{~s}^{1}\right)$ for $\mathrm{Ti}$ and $\left(2 \mathrm{~s}^{2} 2 \mathrm{p}^{1}\right)$ for $\mathrm{B}$. Lattice constants and internal atomic positions of $\mathrm{TiB}, \mathrm{TiB}_{2}$, and $\mathrm{Ti}_{3} \mathrm{~B}_{4}$ unit cells were fully optimized with convergence criteria for the residual Hellman-Feynmann (HF) forces and the system's total energy of $10^{-5} \mathrm{eV} \AA^{-1}$ and $10^{-7} \mathrm{eV}$, respectively. The Brillouin zones of $\mathrm{TiB}, \mathrm{TiB}_{2}$, and $\mathrm{Ti}_{3} \mathrm{~B}_{4}$ were sampled using, respectively, 54, 96, and 50 irreducible $\mathbf{k}$-points generated according to the Monkhorst-Pack scheme. Phonon calculations were performed within the direct method approach $[18,19]$ and harmonic approximation. The HF forces were obtained by displacing the symmetry nonequivalent $\mathrm{Ti}$ and $\mathrm{B}$ atoms from their equilibrium positions by $\pm 0.02 \AA$ in the supercells containing 64 atoms $(\mathrm{TiB}), 92$ atoms $\left(\mathrm{TiB}_{2}\right)$, and 112 atoms $\left(\mathrm{Ti}_{3} \mathrm{~B}_{4}\right)$. The HF forces were calculated with reduced number of k-points. The total number of calculated displacements amounted to 12 for $\mathrm{TiB}, 6$ for $\mathrm{TiB}_{2}$, and 24 for $\mathrm{Ti}_{3} \mathrm{~B}_{4}$. Peak intensities of the nonresonant Raman spectrum (in Stokes process) were calculated from the well-known expression [20]: $I \propto\left|\mathbf{e}_{\mathbf{s}} \mathbf{R e}_{\mathbf{i}}\right|^{2} \omega^{-1}(n+1)$, where $(n+1)$ is the population factor for Stokes scattering with $n=\left[\exp \left(\hbar \omega / k_{B} T\right)-1\right]^{-1}$ denoting the Bose-Einstein thermal factor, $\mathbf{e}_{\mathbf{i}}\left(\mathbf{e}_{\mathbf{s}}\right)$ is the polarization of the incident (scattered) radiation, and $\mathbf{R}$ is the Raman susceptibility tensor. The components of $\mathbf{R}$ tensor $\left(\alpha_{i j}\right)$ were determined from derivatives of the electric polarizability tensor over the atomic displacements [19, 21, 22]. The electric polarizabilities were calculated within the linearresponse method [23] and for each symmetry nonequivalent atom was displaced from its equilibrium position by $\pm 0.01 \AA$. Details of calculations can also be found elsewhere [24, 25]. We also note that anharmonic effects leading to changes in phonon frequencies and reflected by shifts of the Raman peaks' positions have been neglected. This is mainly because our measurements are performed at room temperature, where the effects related to the thermal expansion of compounds from the Ti-B system are negligible. Also, the effect of anharmonicity on the widths of Raman peaks is not considered in the present work. Thus, the Raman peaks are simulated by Lorentzian functions with artificial FWHMs corresponding to energy resolution of the Raman spectrometer used in our studies.

\section{Results and Discussion}

3.1. Structural Properties. Titanium monoboride (TiB) crystallizes in the orthorhombic FeB structure with the space group Pnma (no. 62) [26], where both $\mathrm{Ti}$ and $\mathrm{B}$ atoms occupy (4c) lattice sites. Its primitive unit cell consists of 8 atoms ( 4 formula units). The main building block of TiB is the trigonal prism with the $\mathrm{B}$ atom at the center and the $\mathrm{Ti}$ atoms in corners. The transverse stacking of the trigonal prisms in columnar arrays leads to a zig-zag chain of B atoms along the [010] direction, as schematically shown in Figure 1.

Titanium diboride $\left(\mathrm{TiB}_{2}\right)$ has hexagonal, layered structure of $\mathrm{AlB}_{2}$-type (space group $P 6 / \mathrm{mmm}$, no. 191) with $\mathrm{Ti}$ and $\mathrm{B}$ atoms located, respectively, at $(1 a)$ and $(2 d)$ Wyckoff positions [27]. The primitive unit cell of $\mathrm{TiB}_{2}$ consists of 3 atoms ( 1 formula unit). The $\mathrm{TiB}_{2}$ crystal structure is presented in Figure 2. Each Ti atom is surrounded by 12 equidistant $\mathrm{B}$ atoms, whereas each $\mathrm{B}$ atom has $3 \mathrm{~B}$ atoms at a short distance and $6 \mathrm{Ti}$ atoms at a much longer distance. The B-sublattice 

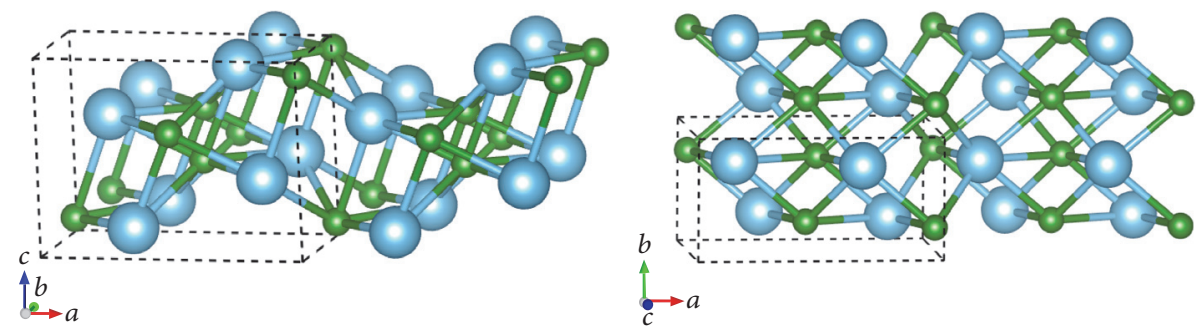

Figure 1: The $2 \times 2 \times 1$ supercells of TiB. Blue and green balls denote Ti and B atoms, respectively. Dashed box represents the unit cell of TiB crystal.
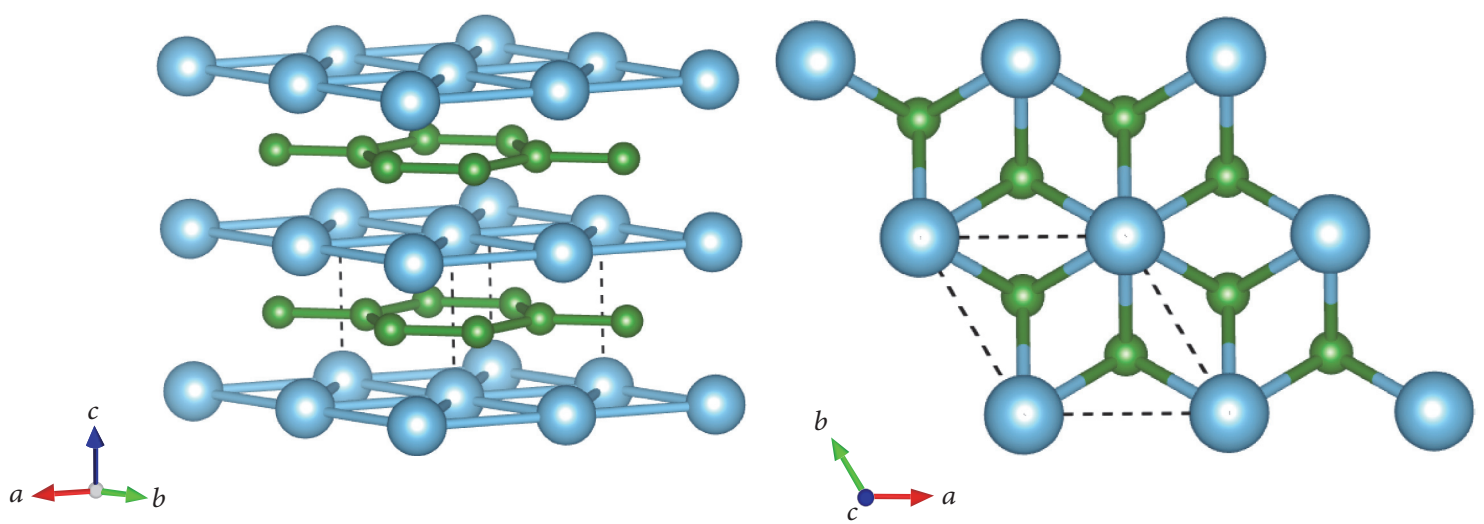

Figure 2: The $2 \times 2 \times 2$ supercell of $\mathrm{TiB}_{2}$. Blue and green balls denote Ti and B atoms, respectively. Dashed box represents the unit cell of $\mathrm{TiB}_{2}$ crystal.

resembles that of graphitic carbon. The Ti-sublattice is nested in the interstices provided by the B-sublattice.

Typical X-ray diffraction spectra of commercial powder and bulk samples of $\mathrm{TiB}_{2}$ are shown in Figure 3. The XRD patterns of powder and target $\mathrm{TiB}_{2}$ are very similar to each other and the Rietveld analysis confirms that the samples contain titanium diboride as a majority phase with the lattice constants $a_{\text {powder }}=3.0178 \AA, c_{\text {powder }}=3.2160 \AA, a_{\text {target }}=$ $3.0324 \AA$, and $c_{\text {target }}=3.2345 \AA$ which correspond to other experimental studies [27] ( $a=3.0292 \AA$ and $c=3.2284 \AA)$.

The crystal structure of $\mathrm{Ti}_{3} \mathrm{~B}_{4}$ is orthorhombic (space group Immm, no. 71) and isomorphous with that of $\mathrm{Ta}_{3} \mathrm{~B}_{4}$ [28]. There are 2 nonequivalent $B$ atoms at ( $4 i)$ and $(4 j)$ lattice sites. Also Ti atoms reside in 2 different Wyckoff positions, namely, $(2 d)$ and $(4 i)$. Thus, the primitive unit cell of $\mathrm{Ti}_{3} \mathrm{~B}_{4}$ contains 14 atoms. The crystal structure of $\mathrm{Ti}_{3} \mathrm{~B}_{4}$ is displayed in Figure 4.

Parameters of the $\mathrm{TiB}, \mathrm{TiB}_{2}$, and $\mathrm{Ti}_{3} \mathrm{~B}_{4}$ structures determined at the ground state are summarized in Table 1 , along with the available experimental data for comparison. In general, the calculated structural parameters of the Ti-B compounds remain in very good agreement with results of the previous experiments [26-28]. Therefore, our theoretical bond lengths between boron atoms $(\mathrm{B}-\mathrm{B})$, titanium and boron atoms $(\mathrm{Ti}-\mathrm{B})$, and between titanium atoms $(\mathrm{Ti}-\mathrm{Ti})$, which are collected in Table 2, closely correspond to those observed in experimental studies. In all considered titanium borides, the shortest bond lengths $(\sim 1.8 \AA)$ are found between $\mathrm{B}$ atoms. The Ti-B bonds are much longer $(\sim 2.4 \AA)$ as compared to $\mathrm{B}-\mathrm{B}$ bonds, but shorter than the $\mathrm{Ti}-\mathrm{Ti}$ bonds $(\sim 2.9 \AA)$. The values of interatomic distances reflect the nature of bonding in titanium borides. This has already been discussed in numerous theoretical studies considering the electronic structure of these compounds [7-11]. Results of the present research confirm that the chemical bonding in $\mathrm{TiB}, \mathrm{TiB}_{2}$, and $\mathrm{Ti}_{3} \mathrm{~B}_{4}$ is a mixture between covalent, ionic, and metallic bonding. Strong covalent bonds exist between $\mathrm{B}$ atoms, while mixed metallic-covalent bonds are between $\mathrm{Ti}$ atoms. There is also a mixed ionic-covalent interaction between Ti and B atoms.

3.2. Zone-Center Phonon Modes. The optically active zonecenter phonon modes in $\mathrm{TiB}, \mathrm{TiB}_{2}$, and $\mathrm{Ti}_{3} \mathrm{~B}_{4}$ are either Raman-active (gerade) or infrared-(IR-) active (ungerade) due to the presence of inversion symmetry in these systems. The $\Gamma$-point phonon modes in $\mathrm{TiB}$ can be decomposed into the irreducible representations of the point group $D_{2 h}^{16}$ as follows: $4 A_{g} \oplus 2 A_{u} \oplus 2 B_{1 g} \oplus 4 B_{1 u} \oplus 4 B_{2 g} \oplus 2 B_{2 u} \oplus 2 B_{3 g} \oplus 4 B_{3 u}$. Among them 3 modes $\left(B_{1 u} \oplus B_{2 u} \oplus B_{3 u}\right)$ are lattice translational modes and $A_{u}$ ones are silent (optically inactive). The modes with symmetries $A_{g}, B_{1 g}, B_{2 g}$, and $B_{3 g}$ are Raman-active, whereas modes $B_{1 u}, B_{2 u}$, and $B_{3 u}$ are IR-active. Both Ti and $B$ atoms occupying the $(4 c)$ lattice positions contribute to the Raman and IR-active modes. The optical IR modes of $B_{1 u}$ and $B_{3 u}$ symmetries correspond to the oscillations of the dipole moment within the crystal ac-plane, whereas those of $B_{2 u}$ symmetry to the oscillations parallel the crystal $b$-axis. The $A_{g}$ and $B_{2 g}$ phonons involve vibrations of 


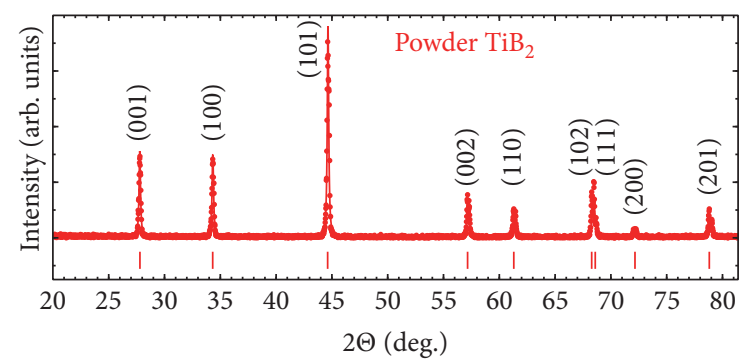

(a)

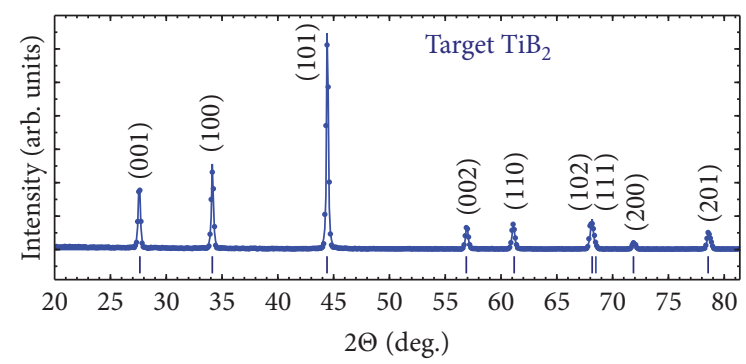

(b)

FIGURE 3: X-ray diffraction spectra of (a) powder (H. C. Starck, Germany) and (b) bulk (target, Goodfellow, UK) samples of titanium diboride $\left(\mathrm{TiB}_{2}\right)$. Experimental data and the Rietveld refinement are represented by symbols and curves, respectively. Small vertical lines indicate positions of the Bragg peaks corresponding to the $\mathrm{TiB}_{2}$ phase. The XRD peaks are indexed according to the reference 04-010-8469 [27].
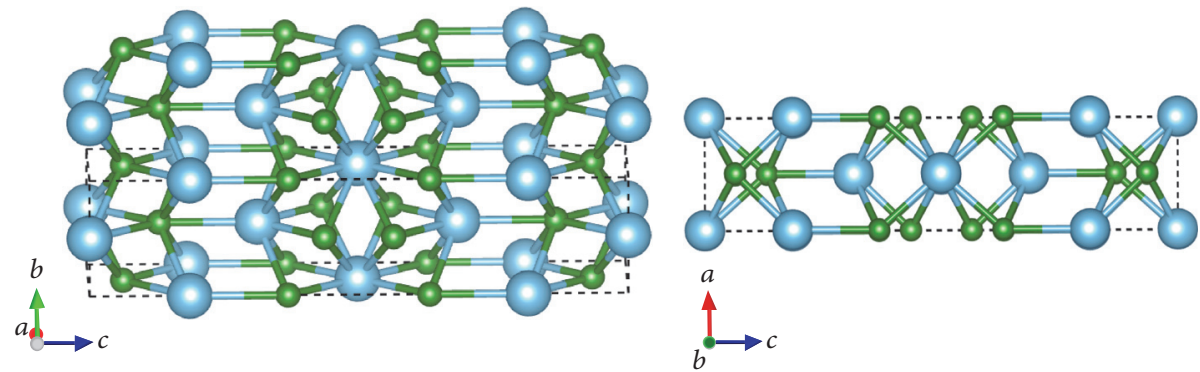

Figure 4: The $1 \times 2 \times 1$ supercell of $\mathrm{Ti}_{3} \mathrm{~B}_{4}$. Blue and green balls denote Ti and $\mathrm{B}$ atoms, respectively. Dashed box represents the unit cell of $\mathrm{Ti}_{3} \mathrm{~B}_{4}$ crystal.

TABLE 1: Structural parameters of $\mathrm{TiB}, \mathrm{TiB}_{2}$, and $\mathrm{Ti}_{3} \mathrm{~B}_{4}$ determined at the ground state and from experiments [26-28].

\begin{tabular}{|c|c|c|}
\hline Parameter & Present & Experiment \\
\hline \multicolumn{2}{|c|}{ TiB (Pnma, no. 62) } & {$[26]$} \\
\hline$a(\AA)$ & 6.1105 & 6.12 \\
\hline$b(\AA)$ & 3.0504 & 3.06 \\
\hline$c(\AA)$ & 4.5623 & 4.56 \\
\hline $\mathrm{Ti}(4 c)$ & $(0.1772,1 / 4,0.1214)$ & $(0.177,1 / 4,0.123)$ \\
\hline $\mathrm{B}(4 c)$ & $(0.0298,1 / 4,0.5985)$ & $(0.029,1 / 4,0.603)$ \\
\hline \multicolumn{2}{|c|}{$\mathrm{TiB}_{2}(P 6 / m m m$, no. 191$)$} & [27] \\
\hline$a(\AA)$ & 3.0336 & 3.0292 \\
\hline$c(\AA)$ & 3.2261 & 3.2284 \\
\hline $\operatorname{Ti}(1 a)$ & $(0.0,0.0,0.0)$ & $(0.0,0.0,0.0)$ \\
\hline $\mathrm{B}(2 d)$ & $(1 / 3,2 / 3,1 / 2)$ & $(1 / 3,2 / 3,1 / 2)$ \\
\hline \multicolumn{2}{|c|}{$\mathrm{Ti}_{3} \mathrm{~B}_{4}($ Immm, no. 71$)$} & {$[28]$} \\
\hline$a(\AA)$ & 3.2596 & 3.259 \\
\hline$b(\AA)$ & 13.7374 & 13.737 \\
\hline$c(\AA)$ & 3.0389 & 3.036 \\
\hline $\mathrm{Ti}_{1}(2 d)$ & $(1 / 2,1 / 2,0.0)$ & $(1 / 2,1 / 2,0.0)$ \\
\hline $\mathrm{Ti}_{2}(4 i)$ & $(0.0,0.1858,0.0)$ & $(0.0,0.18,0.0)$ \\
\hline $\mathrm{B}_{1}(4 i)$ & $(0.0,0.3684,0.0)$ & $(0.0,0.37,0.0)$ \\
\hline$B_{2}(4 j)$ & $(0.0,0.4356,1 / 2)$ & $(0.0,0.44,1 / 2)$ \\
\hline
\end{tabular}

the $\mathrm{Ti}$ - and $\mathrm{B}$-sublattices within the ac-plane, while the $B_{1 g}$ and $B_{3 g}$ phonons arise from atomic vibrations along the $b$-axis. The frequencies of the Raman and IR-active
TABLE 2: Interatomic distances (in $\AA$ ) for titanium borides.

\begin{tabular}{lccc}
\hline Compound & $\mathrm{B}-\mathrm{B}$ & $\mathrm{Ti}-\mathrm{B}$ & $\mathrm{Ti}-\mathrm{Ti}$ \\
\hline $\mathrm{TiB}$ & 1.81 & 2.35 & 2.87 \\
$\mathrm{TiB}_{2}$ & 1.75 & 2.38 & 3.03 \\
& $1.77\left(\mathrm{~B}_{2}-\mathrm{B}_{2}\right)$ & $2.33\left(\mathrm{Ti}_{2}-\mathrm{B}_{2}\right)$ & $2.84\left(\mathrm{Ti}_{2}-\mathrm{Ti}_{2}\right)$ \\
& $1.78\left(\mathrm{~B}_{1}-\mathrm{B}_{1}\right)$ & $2.35\left(\mathrm{Ti}_{2}-\mathrm{B}_{1}\right)$ & $2.97\left(\mathrm{Ti}_{1}-\mathrm{Ti}_{2}\right)$ \\
$\mathrm{Ti}_{3} \mathrm{~B}_{4}$ & $3.04\left(\mathrm{~B}_{1}-\mathrm{B}_{2}\right)$ & $2.40\left(\mathrm{Ti}_{1}-\mathrm{B}_{2}\right)$ & $3.04\left(\mathrm{Ti}_{1}-\mathrm{Ti}_{1}\right)$ \\
& & $2.43\left(\mathrm{Ti}_{1}-\mathrm{B}_{1}\right)$ & \\
& &
\end{tabular}

phonon modes predicted by our calculations for $\mathrm{TiB}$ are listed in Table 3. The silent $A_{u}$ modes are found at 280.2 and $453.1 \mathrm{~cm}^{-1}$. The IR modes gather into 2 bands with lower-frequency band located at $\sim 250 \mathrm{~cm}^{-1}$ and the higherfrequency band extending from about 470 to $560 \mathrm{~cm}^{-1}$. Similarly, the Raman modes are also concentrated within 2 bands. The lower-frequency band ranges from about 260 to $350 \mathrm{~cm}^{-1}$ and the higher-frequency one from 570 to $780 \mathrm{~cm}^{-1}$.

Phonons at the Brillouin zone center of the $\mathrm{TiB}_{2}$ structure can be classified according to the irreducible representations of the point group $D_{6 h}^{1}$ as follows: $A_{2 u} \oplus B_{1 g} \oplus 2 E_{2 g}^{(2)} \oplus 2 E_{1 u}^{(2)}$. The modes with $A_{2 u}$ and $E_{1 u}$ symmetries are IR-active, the modes of $E_{2 g}$ symmetry are Raman-active, and the $B_{1 g}$ mode is silent. Modes $E_{2 g}$ and $E_{1 u}$ remain doubly degenerate. The $A_{2 u} \oplus E_{1 u}^{(2)}$ phonons constitute lattice translational modes. The IR-active $A_{2 u}$ and $E_{1 u}$ modes are related to the dipole moment oscillations perpendicular and parallel to the crystal hexagonal plane, respectively. In the Raman-active $E_{2 g}$ modes 
TABLE 3: Frequencies of the Raman and IR-active phonon modes in TiB. Units: $\mathrm{cm}^{-1}$.

\begin{tabular}{lcc}
\hline Mode symmetry & Raman & Infrared \\
\hline$B_{1 u}$ & & 245 \\
$B_{3 u}$ & 259 & \\
$A_{g}$ & 272 & \\
$B_{3 g}$ & 293 & \\
$B_{2 g}$ & 299 & \\
$B_{1 g}$ & 305 & \\
$A_{g}$ & 347 & 468 \\
$B_{2 g}$ & & 494 \\
$B_{3 u}$ & & 499 \\
$B_{1 u}$ & & 542 \\
$B_{2 u}$ & & 564 \\
$B_{3 u}$ & & \\
$B_{1 u}$ & 570 & \\
$A_{g}$ & 607 & \\
$B_{2 g}$ & 634 & \\
$B_{2 g}$ & 639 & \\
$A_{g}$ & 760 & \\
$B_{1 g}$ & 780 & \\
$B_{3 g}$ & & \\
\hline
\end{tabular}

the $\mathrm{Ti}$ atoms are at rest, and hence these modes are only associated with the $\mathrm{B}$ atoms vibrating within the hexagonal plane. The $E_{2 g}$ Raman phonon appears at $883.1 \mathrm{~cm}^{-1}$ and the infrared $E_{1 u}$ and $A_{2 u}$ phonons have frequencies of $515.1 \mathrm{~cm}^{-1}$ and $521.5 \mathrm{~cm}^{-1}$, respectively. The calculated frequency of the silent $B_{1 \mathrm{~g}}$ amounts to $557.9 \mathrm{~cm}^{-1}$. The frequencies of the Raman and infrared modes in $\mathrm{TiB}_{2}$ crystal determined in the present DFT studies closely correlated with those obtained previously $[12,13]$.

The $\Gamma$-point phonon modes in $\mathrm{Ti}_{3} \mathrm{~B}_{4}$ can be decomposed into the irreducible representations of the point group $D_{2 h}^{25}$ in the following way: $3 A_{g} \oplus 3 B_{1 g} \oplus 3 B_{3 g} \oplus 4 B_{1 u} \oplus 4 B_{2 u} \oplus$ $4 B_{3 u}$, where the Raman modes have symmetries of $A_{g}, B_{2 g}$, and $B_{3 g}$. The $B_{1 u}, B_{2 u}$, and $B_{3 u}$ modes are infrared-active. There are 3 acoustic modes constituted by the IR phonons $\left(\Gamma_{\text {acoustic }}=B_{1 u} \oplus B_{2 u} \oplus B_{3 u}\right)$. The IR-active $B_{1 u}, B_{2 u}$, and $B_{3 u}$ are associated with the oscillations of the dipole moment along the crystallographic $c, b$, and $a$ axes, respectively. The $\mathrm{Ti}_{1}$ atoms residing in $(2 d)$ sites do not contribute to the Raman modes. Therefore, the $A_{g}, B_{2 g}$, and $B_{3 g}$ phonons results from the displacements of $\mathrm{Ti}_{2}, \mathrm{~B}_{1}$, and $\mathrm{B}_{2}$ atoms along the $c, a$, and $b$ axes of the $\mathrm{Ti}_{3} \mathrm{~B}_{4}$ crystal. Respective frequencies of the Raman and IR modes are collected in Table 4.

3.3. Raman Spectra. The Raman tensors of the $A_{g}, B_{1 g}, B_{2 g}$, and $B_{3 g}$ phonon modes in TiB have the following nonzero components:

$$
\begin{aligned}
A_{g}: \quad \alpha_{x x} & =a, \\
\alpha_{y y} & =b, \\
\alpha_{z z} & =c,
\end{aligned}
$$

TABLE 4: Frequencies of the Raman and IR-active phonon modes in $\mathrm{Ti}_{3} \mathrm{~B}_{4}$. Units: $\mathrm{cm}^{-1}$.

\begin{tabular}{lcc}
\hline Mode symmetry & Raman & Infrared \\
\hline$B_{3 g}$ & 249 & \\
$B_{1 g}$ & 263 & 277 \\
$B_{1 u}$ & & 287 \\
$B_{3 u}$ & & 313 \\
$B_{2 u}$ & 323 & \\
$A_{g}$ & & 483 \\
$B_{2 u}$ & & 488 \\
$B_{1 u}$ & 499 & \\
$B_{3 g}$ & 504 & 528 \\
$B_{1 g}$ & & \\
$B_{3 u}$ & 550 & 556 \\
$A_{g}$ & & \\
$B_{3 u}$ & 574 & 815 \\
$B_{1 g}$ & & \\
$B_{2 u}$ & & \\
$B_{1 u}$ & 829 & \\
$B_{3 g}$ & 835 & \\
$A_{g}$ & &
\end{tabular}

$$
\begin{aligned}
& B_{1 g}: \quad \alpha_{x y}=\alpha_{y x}=d, \\
& B_{2 g}: \quad \alpha_{x z}=\alpha_{z x}=e, \\
& B_{3 g}: \quad \alpha_{y z}=\alpha_{z y}=f
\end{aligned}
$$

and the polarization selection rules [29] for the point group $D_{6 h}^{1}$ allow the polarized Raman scattering experiments to determine phonons having particular symmetries. In the backscattering geometry, where the wave vector of incident $\left(\mathbf{k}_{\mathbf{i}}\right)$ and scattered $\left(\mathbf{k}_{\mathbf{s}}\right)$ radiations remain antiparallel, the modes of $A_{g}$ symmetry can be measured, for example, at $Z(X X) \bar{Z}$ scattering configuration (in Porto's notation). In order to observe the $B_{1 g}, B_{2 g}$, and $B_{3 g}$ modes one needs to apply the $Z(X Y) \bar{Z}, Y(X Z) \bar{Y}$, and $X(Y Z) \bar{X}$ scattering geometries, respectively. The polarized backscattering Raman spectra at scattering configurations outlined above are shown in Figure 5. One notes that not all Raman-active modes of TiB are intense enough to be experimentally observed.

The Raman spectrum of $\mathrm{TiB}_{2}$ single crystal is characterized by a single peak due to the mode of $E_{2 g}$ symmetry, which can be detected at $Z(X Y) \bar{Z}$ scattering geometry. The corresponding Raman tensor of the doubly degenerate $E_{2 g}$ phonon mode has the following form:

$$
\begin{aligned}
E_{2 g}^{(1)}: & \alpha_{x x}=d, \\
& \alpha_{y y}=-d, \\
E_{2 g}^{(2)}: & \alpha_{x y}=\alpha_{y x}=-d .
\end{aligned}
$$

The Raman tensors of the $A_{g}, B_{1 g}$, and $B_{3 g}$ modes in $\mathrm{Ti}_{3} \mathrm{~B}_{4}$ crystal are defined in the same manner as for the TiB 


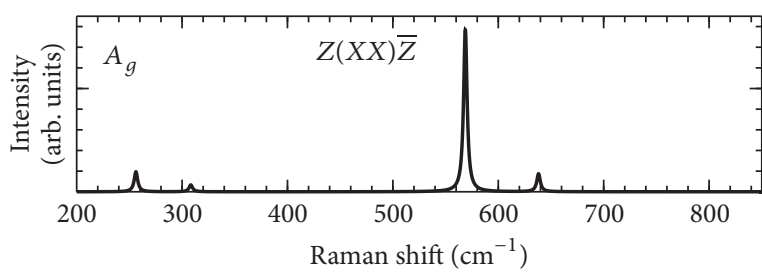

(a)

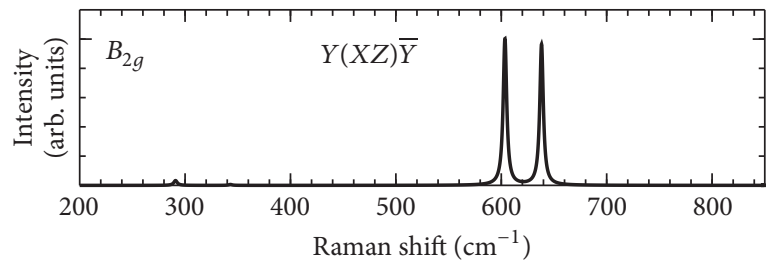

(c)

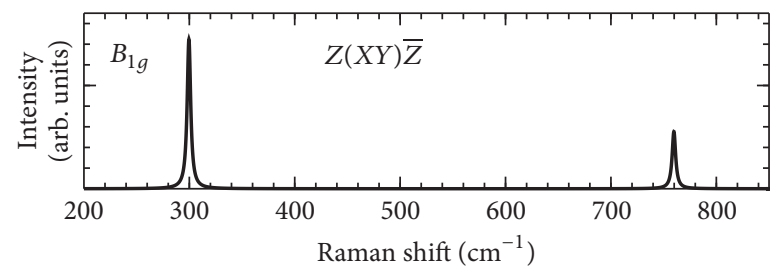

(b)

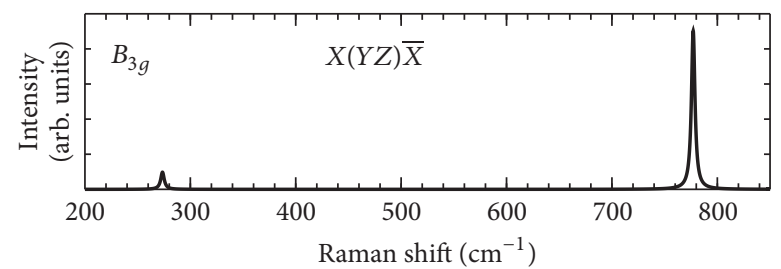

(d)

FIGURE 5: Backscattering Raman spectra of TiB crystal calculated at scattering geometries (a) $Z(X X) \bar{Z}$, (b) $Z(X Y) \bar{Z}$, (c) $Y(Z X) \bar{Y}$, and (d) $X(Y Z) \bar{X}$. Spectra are simulated at $300 \mathrm{~K}$ and with laser excitation wavelength of $532 \mathrm{~nm}$. Peaks are represented by Lorentzian functions with artificial FWHMs of $2 \mathrm{~cm}^{-1}$.

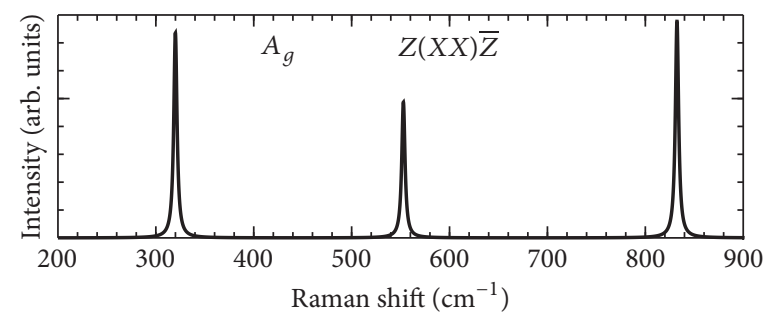

(a)

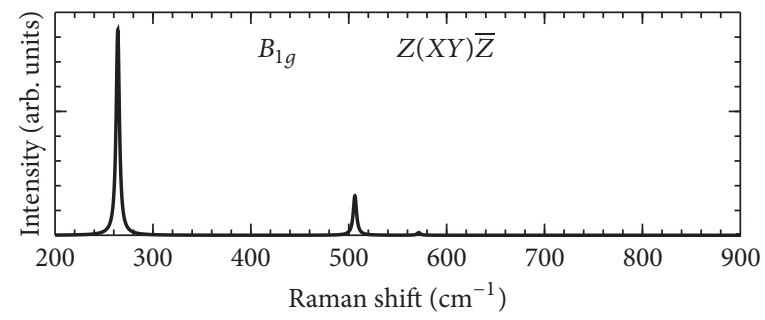

(b)

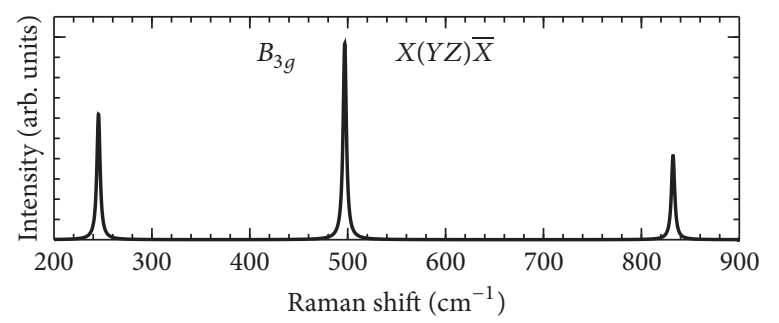

(c)

FIgure 6: Backscattering Raman spectra of $\mathrm{Ti}_{3} \mathrm{~B}_{4}$ crystal calculated at scattering geometries (a) $Z(X X) \bar{Z}$, (b) $Z(X Y) \bar{Z}$, and (c) $X(Y Z) \bar{X}$. Spectra are simulated at $300 \mathrm{~K}$ and with laser excitation wavelength of $532 \mathrm{~nm}$. Peaks are represented by Lorentzian functions with artificial FWHMs of $2 \mathrm{~cm}^{-1}$.

crystal (see (1)). Therefore, one determines the $A_{g}, B_{1 g}$, and $B_{3 g}$ phonons in $\mathrm{Ti}_{3} \mathrm{~B}_{4}$ by using the same scattering geometries as those given for orthorhombic TiB crystal. The resulting Raman spectra are presented in Figure 6.

In majority of cases, experimental characterization of the Ti-B material by using the Raman spectroscopy is based on measurements performed on powder samples, and hence the resulting spectra of polycrystalline materials may differ from those for single crystals. Indeed, the simulated unpolarized Raman spectra in backscattering geometry of TiB, $\mathrm{TiB}_{2}$, and $\mathrm{Ti}_{3} \mathrm{~B}_{4}$ polycrystals, which are shown in Figure 7, remain quite distinct from the polarized spectra of the respective single crystals given in Figures 5 and 6. First of all, not all Raman-active modes are observed due to their weak intensities. The peaks of $\mathrm{TiB}$ and $\mathrm{Ti}_{3} \mathrm{~B}_{4}$ polycrystals originate from phonons of the $A_{g}$ symmetry. Therefore, the unpolarized Raman spectrum of multiphase $\mathrm{Ti}-\mathrm{B}$ system may consist of three bands. The low-frequency $\left(240-360 \mathrm{~cm}^{-1}\right)$ and middle-frequency $\left(520-680 \mathrm{~cm}^{-1}\right)$ bands are expected to be dominated by the modes of $\mathrm{TiB}$ and $\mathrm{Ti}_{3} \mathrm{~B}_{4}$ phases, whereas the high-frequency band $\left(800-900 \mathrm{~cm}^{-1}\right)$ is expected to be dominated by the modes of $\mathrm{TiB}_{2}$ and $\mathrm{Ti}_{3} \mathrm{~B}_{4}$ phases.

According to the group symmetry analysis, the $\mathrm{TiB}_{2}$ compound exhibits a single doubly degenerate Raman-active mode of $E_{2 g}$ symmetry, which should be revealed by the Raman spectra of either a single crystal or polycrystalline 


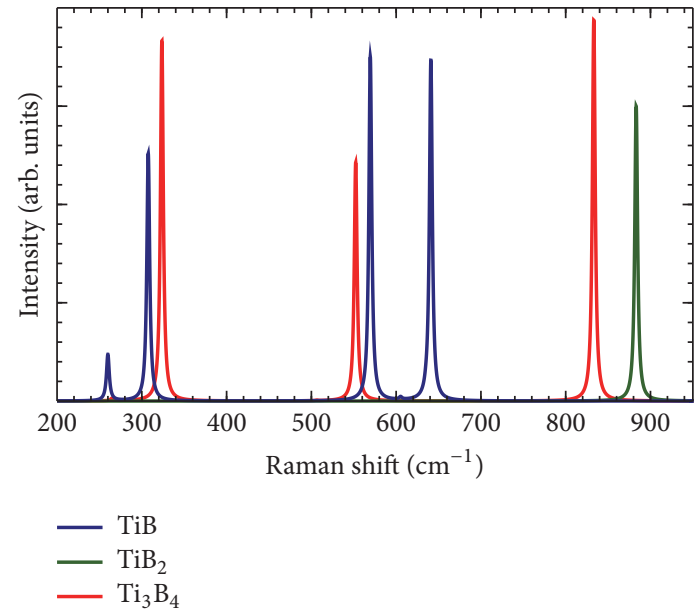

Figure 7: Unpolarized Raman spectra of $\mathrm{TiB}, \mathrm{TiB}_{2}$, and $\mathrm{Ti}_{3} \mathrm{~B}_{4}$ polycrystals calculated at backscattering geometries. Spectra are simulated at $300 \mathrm{~K}$ and with laser excitation wavelength of $532 \mathrm{~nm}$. Peaks are represented by Lorentzian functions with artificial FWHMs of $2 \mathrm{~cm}^{-1}$.

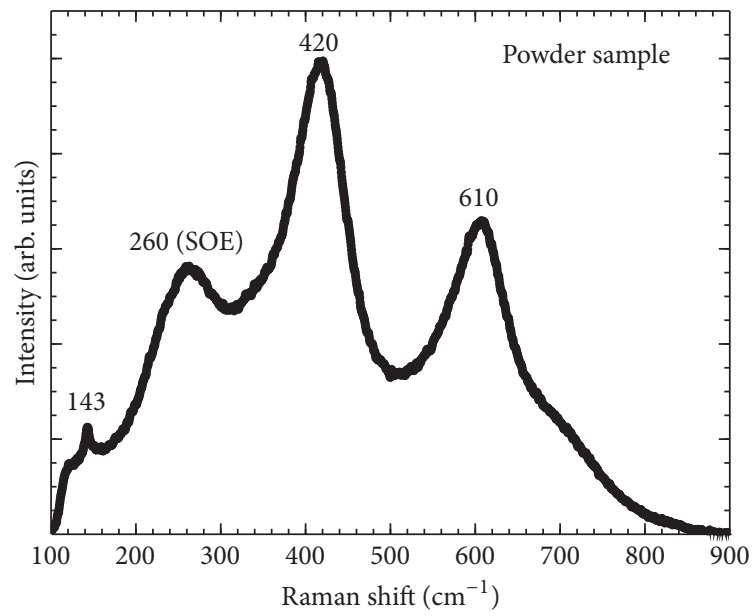

Figure 8: Experimental Raman spectrum of commercial $\mathrm{TiB}_{2}$ powder (H. C. Starck, Germany) measured at room temperature with laser excitation wavelength of $532 \mathrm{~nm}$.

samples. However, a typical Raman spectrum of $\mathrm{TiB}_{2}$ commercial powder sample shows more rich experimental pattern than that predicted theoretically. Such spectrum is presented in Figure 8. It shows a small intensity peak at $143 \mathrm{~cm}^{-1}$ and broad high-intensity peaks at 260,420 , and $610 \mathrm{~cm}^{-1}$. These peaks are characteristic for rutile titanium dioxide $\left(\mathrm{TiO}_{2}\right)$ phase, whose vibrational spectrum has 4 vibrational bands centered around $145 \mathrm{~cm}^{-1}\left(B_{1 g}\right)$, $445 \mathrm{~cm}^{-1}\left(E_{g}\right), 610 \mathrm{~cm}^{-1}\left(A_{1 g}\right)$, and $240 \mathrm{~cm}^{-1}$ for secondorder scattering effect $(\mathrm{SOE})[30,31]$. We note that similar spectrum was also obtained for commercial powder $\mathrm{TiB}_{2}$, although with slightly different positions of the Raman peaks $\left(260,409\right.$, and $\left.598 \mathrm{~cm}^{-1}\right)$, and it was assigned to the anatase phase of $\mathrm{TiO}_{2}$ [32]. Our spectrum is unlikely to represent powder anatase $\mathrm{TiO}_{2}$, as such spectrum usually shows 5 Raman modes centered around $144 \mathrm{~cm}^{-1}\left(E_{g}\right)$,

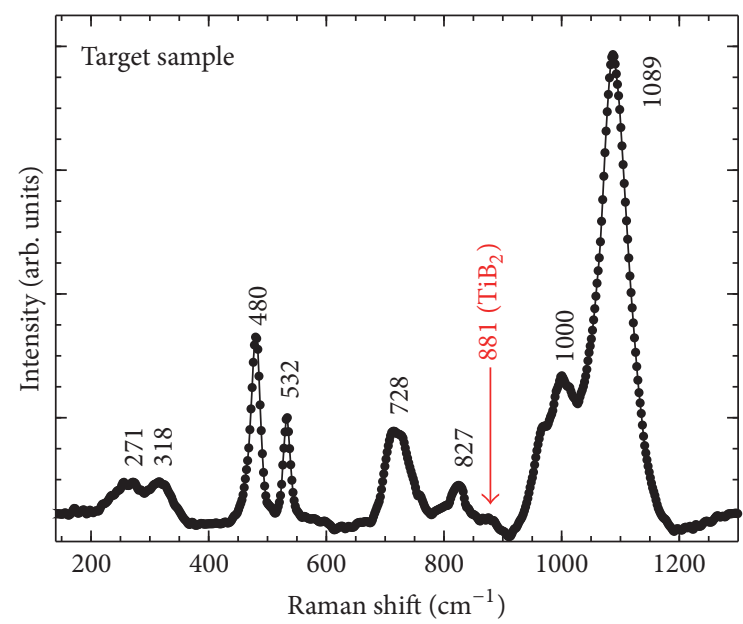

FIgURE 9: Experimental Raman spectrum of commercial $\mathrm{TiB}_{2}$ target (Goodfellow, UK) measured at room temperature with laser excitation wavelength of $532 \mathrm{~nm}$.

$196 \mathrm{~cm}^{-1}\left(E_{g}\right), 394 \mathrm{~cm}^{-1}\left(B_{1 g}\right), 516 \mathrm{~cm}^{-1}\left(B_{1 g}+A_{1 g}\right)$, and $638 \mathrm{~cm}^{-1}\left(E_{g}\right)[30,33,34]$. Moreover, the characteristic feature of anatase spectrum is the high-intensity peak at $144 \mathrm{~cm}^{-1}$ which dominates over the remaining peaks having comparably smaller intensities. In addition, the highfrequency range of our spectrum reveals two quite intense peaks at about 1360 and $1570 \mathrm{~cm}^{-1}$ indicating the presence of graphitic carbon with $s p^{2}$ and $s p^{3}$ bonds [35]. Nevertheless, we confirm that even a small amount of contaminating phase, such as $\mathrm{TiO}_{2}$ and unreacted carbon, being by-products of the carbothermal reduction process employed to fabricate $\mathrm{TiB}_{2}$ powder according to the following reaction [36]:

$$
\mathrm{TiO}_{2}+\mathrm{B}_{2} \mathrm{O}_{3}+5 \mathrm{C} \longrightarrow \mathrm{TiB}_{2}+5 \mathrm{CO} \uparrow
$$

can prevail in the Raman spectrum of commercial $\mathrm{TiB}_{2}$ powder.

$\mathrm{TiB}_{2}$ can also be prepared by reduction of $\mathrm{TiO}_{2}$ by boron carbide $\left(\mathrm{B}_{4} \mathrm{C}\right)$ and carbon as follows [37]:

$$
2 \mathrm{TiO}_{2}+\mathrm{B}_{4} \mathrm{C}+3 \mathrm{C} \longrightarrow 2 \mathrm{TiB}_{2}+4 \mathrm{CO} \uparrow
$$

The above procedure is frequently applied to produce commercial targets of $\mathrm{TiB}_{2}$. Thus, the Raman spectrum of such target usually shows a dominant contribution from the byproducts of synthesis and consolidation reactions, as shown in Figure 9. Here, the main peaks appearing at 271, 318, 480, $532,728,827,1000$, and $1089 \mathrm{~cm}^{-1}$ are associated with the amorphous $\mathrm{B}_{4} \mathrm{C}$ phase which displays characteristic Raman bands at $270,320,481,531,728,830,1000$, and $1088 \mathrm{~cm}^{-1}$ [38-40]. Additionally one detects a weak feature at about $970 \mathrm{~cm}^{-1}$ which is also visible in the previously measured spectra of crystalline and amorphous boron carbide [39, 40]. Besides, our Raman spectrum of target sample reveals a peak at $881 \mathrm{~cm}^{-1}$ being an evidence of the presence of $\mathrm{TiB}_{2}$ phase, for which a Raman peak was predicted by our DFT calculations at $883 \mathrm{~cm}^{-1}$. 


\section{Summary and Conclusions}

The present work reports on experimental Raman spectra of commercially available powder and bulk samples of titanium diboride. It is shown that micro-Raman spectroscopy enables identification of impurity phases contained in the samples even though their concentration remains below 1 wt.\%. Detailed analysis uncover contamination of commercial $\mathrm{TiB}_{2}$ powder and bulk samples by $\mathrm{TiO}_{2}$ and $\mathrm{B}_{4} \mathrm{C}$ phases, respectively, which are the by-products of chemical reactions applied to produce samples. Additionally, the graphitic carbon is identified as a fingerprint of sintering aids used in production process of bulk $\mathrm{TiB}_{2}$. Vibrational properties of titanium borides $\left(\mathrm{TiB}, \mathrm{TiB}_{2}\right.$, and $\left.\mathrm{Ti}_{3} \mathrm{~B}_{4}\right)$, in particular, positions and intensities of the Raman-active phonons, were gained from theoretical approach based on the DFT method. Theoretical Raman spectra were simulated at conditions close to those encountered in experiments and for ideal crystals, namely, free of defects and residual stresses which are always present in real samples due to their preparation procedure. Thus, our $a b$ initio results can serve not only as a guide for interpretation of experimental Raman spectra or symmetry mode assignment in particular titanium borides, but also for estimation of effects connected with macrostresses and their influence on positions and intensities of the measured Raman peaks.

\section{Competing Interests}

The authors declare that there are no competing interests regarding the publication of this paper.

\section{Acknowledgments}

Interdisciplinary Center for Mathematical and Computational Modeling (ICM), Warsaw University, Poland, is acknowledged for providing the computer facilities under Grant no. G28-12.

\section{References}

[1] J. L. Murray, P. K. Liao, and K. E. Spear, "The B-Ti (BoronTitanium) system," Bulletin of Alloy Phase Diagrams, vol. 7, no. 6, pp. 550-555, 1986.

[2] V. I. Matkovich, Boron and Refractory Borides, Springer, Berlin, Germany, 1977.

[3] R. G. Munro, "Material properties of titanium diboride," Journal of Research of the National Institute of Standards and Technology, vol. 105, no. 5, pp. 709-720, 2000.

[4] M. Gu, C. Huang, B. Zou, and B. Liu, "Effect of (Ni, Mo) and TiN on the microstructure and mechanical properties of $\mathrm{TiB}_{2}$ ceramic tool materials," Materials Science and Engineering A, vol. 433, no. 1-2, pp. 39-44, 2006.

[5] P. H. Mayrhofer, C. Mitterer, L. Hultman, and H. Clemens, "Microstructural design of hard coatings," Progress in Materials Science, vol. 51, no. 8, pp. 1032-1114, 2006.

[6] A. Twardowska, "On mechanical and friction-wear properties of TiBx coated alumina sinters," Mechanik, no. 5-6, pp. 492-493, 2016.
[7] D.-C. Tian and X.-B. Wang, "Electronic structure and equation of state of TiB2," Journal of Physics: Condensed Matter, vol. 4, no. 45, pp. 8765-8772, 1992.

[8] P. Vajeeston, P. Ravindran, C. Ravi, and R. Asokamani, "Electronic structure, bonding, and ground-state properties of $\mathrm{AlB}_{2}$ type transition-metal diborides," Physical Review B, vol. 63, no. 4, Article ID 045115, 12 pages, 2001.

[9] K. B. Panda and K. S. R. Chandran, "First principles determination of elastic constants and chemical bonding of titanium boride (TiB) on the basis of density functional theory," Acta Materialia, vol. 54, no. 6, pp. 1641-1657, 2006.

[10] L. Sun, Y. Gao, B. Xiao, Y. Li, and G. Wang, "Anisotropic elastic and thermal properties of titanium borides by first-principles calculations," Journal of Alloys and Compounds, vol. 579, pp. 457-467, 2013.

[11] G. Wang, Y. Li, Y. Gao, Y. Cheng, and S. Ma, “Theoretical study of structural, mechanical, thermal and electronic properties of $\mathrm{Ti}_{3} \mathrm{~B}_{4}$ with $\mathrm{Ta}_{3} \mathrm{~B}_{4}$ structure under high pressure," Computational Materials Science, vol. 104, pp. 29-34, 2015.

[12] R. Heid, B. Renker, H. Schober, P. Adelmann, D. Ernst, and K.P. Bohnen, "Lattice dynamics and electron-phonon coupling in transition-metal diborides," Physical Review B, vol. 67, no. 18, Article ID 180510, pp. 1-4, 2003.

[13] E. Deligoz, K. Colakoglu, and Y. Ciftci, "Lattice dynamical properties of $\mathrm{SCB}_{2}, \mathrm{TiB}_{2}$, and $\mathrm{VB}_{2}$ compounds," Solid State Communications, vol. 149, no. 41-42, pp. 1843-1848, 2009.

[14] G. Kresse and J. Furthmüller, "Efficient iterative schemes for $a b$ initio total-energy calculations using a plane-wave basis set," Physical Review B, vol. 54, no. 16, pp. 11169-11186, 1996.

[15] G. Kresse and J. Furthmüller, "Efficiency of ab-initio total energy calculations for metals and semiconductors using a plane-wave basis set," Computational Materials Science, vol. 6, no. 1, pp. 15-50, 1996.

[16] J. P. Perdew, K. Burke, and M. Ernzerhof, "Generalized gradient approximation made simple," Physical Review Letters, vol. 77, no. 18, pp. 3865-3868, 1996.

[17] J. P. Perdew, K. Burke, and M. Ernzerhof, "Generalized gradient approximation made simple [Phys. Rev. Lett. 77, 3865 (1996)]," Physical Review Letters, vol. 78, no. 7, p. 1396, 1997.

[18] K. Parlinski, Z. Q. Li, and Y. Kawazoe, "First-principles determination of the soft mode in cubic $\mathrm{ZrO}_{2}$," Physical Review Letters, vol. 78, no. 21, article 4063, 1997.

[19] K. Parlinski, Software PHONON ver. 6.15, Cracow, Poland, 2015.

[20] M. Cardona, Ed., Light Scattering in Solids I, vol. 8 of Topics in Applied Physics, Springer, Berlin, Germany, 1983.

[21] P. Umari, A. Pasquarello, and A. Dal Corso, "Raman scattering intensities in $\alpha$-quartz: a first-principles investigation," Physical Review B, vol. 63, no. 9, Article ID 094305, 2001.

[22] P. Umari, X. Gonze, and A. Pasquarello, "Concentration of small ring structures in vitreous silica from a first-principles analysis of the Raman spectrum," Physical Review Letters, vol. 90, no. 2, Article ID 027401, 2003.

[23] M. Gajdoš, K. Hummer, G. Kresse, J. Furthmüller, and F. Bechstedt, "Linear optical properties in the projector-augmented wave methodology," Physical Review B, vol. 73, no. 4, Article ID 045112, 2006.

[24] U. D. Wdowik, A. Twardowska, and M. Medala-Wasik, "Lattice dynamics of binary and ternary phases in Ti-Si-C system: a combined Raman spectroscopy and density functional theory study," Materials Chemistry and Physics, vol. 168, pp. 58-65, 2015. 
[25] U. D. Wdowik, M. Wasik, and A. Twardowska, "Influence of carbon dopants on the structure, elasticity and lattice dynamics of $\mathrm{Ti}_{5} \mathrm{Si}_{3} \mathrm{C}_{x}$ Nowotny phases," Modelling and Simulation in Materials Science and Engineering, vol. 24, Article ID 025001, 2016.

[26] B. F. Decker and J. S. Kasper, "The crystal structure of TiB," Acta Crystallographica, vol. 7, no. 1, pp. 77-80, 1954.

[27] S. Möhr, H. Müller-Buschbaum, Y. Grim, and H. G. von Schnering, "H-TiO oder $\mathrm{TiB}_{2}$ ??-eine Korrektur," Zeitschrift für Anorganische und Allgemeine Chemie, vol. 622, no. 2, pp. 10351037, 1996.

[28] K. E. Spear, P. Mcdowell, and F. Mcmahon, "Experimental evidence for the existence of the $\mathrm{Ti}_{3} \mathrm{~B}_{4}$ phase," Journal of the American Ceramic Society, vol. 69, no. 1, pp. C-4-C-5, 1986.

[29] R. Loudon, "The Raman effect in crystals," Advances in Physics, vol. 50, no. 7, pp. 813-864, 2001.

[30] O. Frank, M. Zukalova, B. Laskova, J. Kürti, J. Koltai, and L. Kavan, "Raman spectra of titanium dioxide (anatase, rutile) with identified oxygen isotopes $(16,17,18)$, , Physical Chemistry Chemical Physics, vol. 14, no. 42, pp. 14567-14572, 2012.

[31] Y. Zhang, C. X. Harris, P. Wallenmeyer, J. Murowchick, and $\mathrm{X}$. Chen, "Asymmetric lattice vibrational characteristics of rutile $\mathrm{TiO}_{2}$ as revealed by laser power dependent raman spectroscopy," Journal of Physical Chemistry C, vol. 117, no. 45, pp. 24015-24022, 2013.

[32] L'. Bača and N. Stelzer, "Adapting of sol-gel process for preparation of TiB2 powder from low-cost precursors," Journal of the European Ceramic Society, vol. 28, no. 5, pp. 907-911, 2008.

[33] V. Swamy, A. Kuznetsov, L. S. Dubrovinsky, R. A. Caruso, D. G. Shchukin, and B. C. Muddle, "Finite-size and pressure effects on the Raman spectrum of nanocrystalline anatase $\mathrm{TiO}_{2}$," Physical Review B - Condensed Matter and Materials Physics, vol. 71, no. 18, Article ID 184302, 2005.

[34] X. Chen and S. S. Mao, "Titanium dioxide nanomaterials: synthesis, properties, modifications and applications," Chemical Reviews, vol. 107, no. 7, pp. 2891-2959, 2007.

[35] F. Tuinstra and J. L. Koenig, "Raman spectrum of graphite," The Journal of Chemical Physics, vol. 53, no. 3, pp. 1126-1130, 1970.

[36] R. V. Krishnarao and J. Subrahmanyam, "Studies on the formation of $\mathrm{TiB}_{2}$ through carbothermal reduction of $\mathrm{TiO}_{2}$ and $\mathrm{B}_{2} \mathrm{O}_{3}$," Materials Science and Engineering A, vol. 362, no. 1-2, pp. 145-151, 2003.

[37] C. Subramanian, T. S. R. C. Murthy, and A. K. Suri, "Synthesis and consolidation of titanium diboride," International Journal of Refractory Metals and Hard Materials, vol. 25, no. 4, pp. 345350, 2007.

[38] X. Q. Yan, W. J. Li, T. Goto, and M. W. Chen, "Raman spectroscopy of pressure-induced amorphous boron carbide," Applied Physics Letters, vol. 88, no. 13, Article ID 131905, 2006.

[39] J. Guo, L. Zhang, T. Fujita, T. Goto, and M. Chen, "Pressureinduced depolarization and resonance in Raman scattering of single-crystalline boron carbide," Physical Review B, vol. 81, no. 6, Article ID 060102, 2010.

[40] K. M. Reddy, P. Liu, A. Hirata, T. Fujita, and M. W. Chen, "Atomic structure of amorphous shear bands in boron carbide," Nature Communications, vol. 4, article 2483, 2013. 

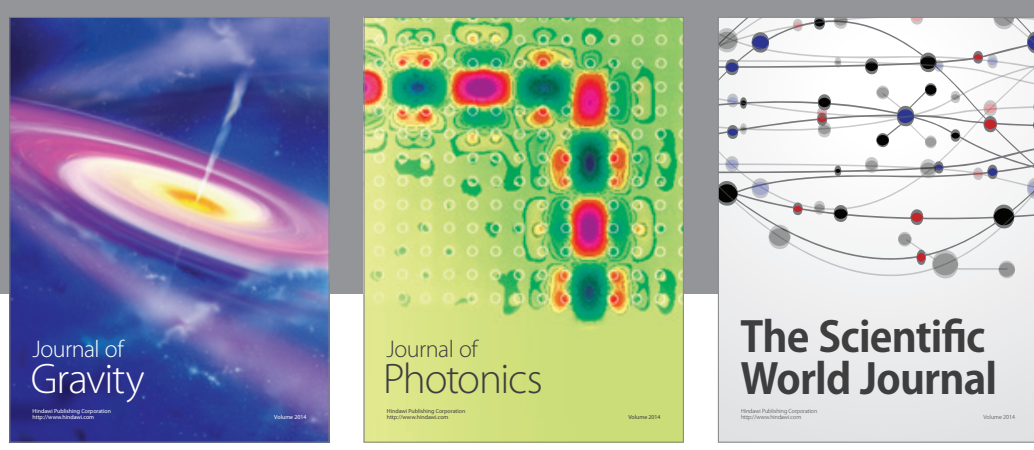

The Scientific World Journal
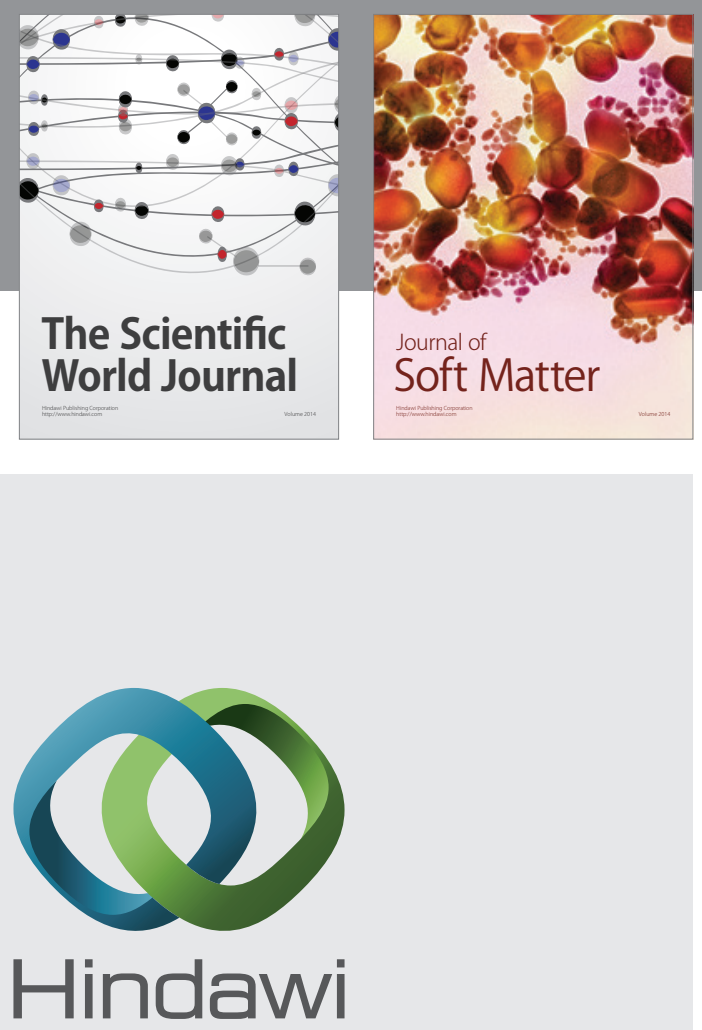

Submit your manuscripts at

https://www.hindawi.com
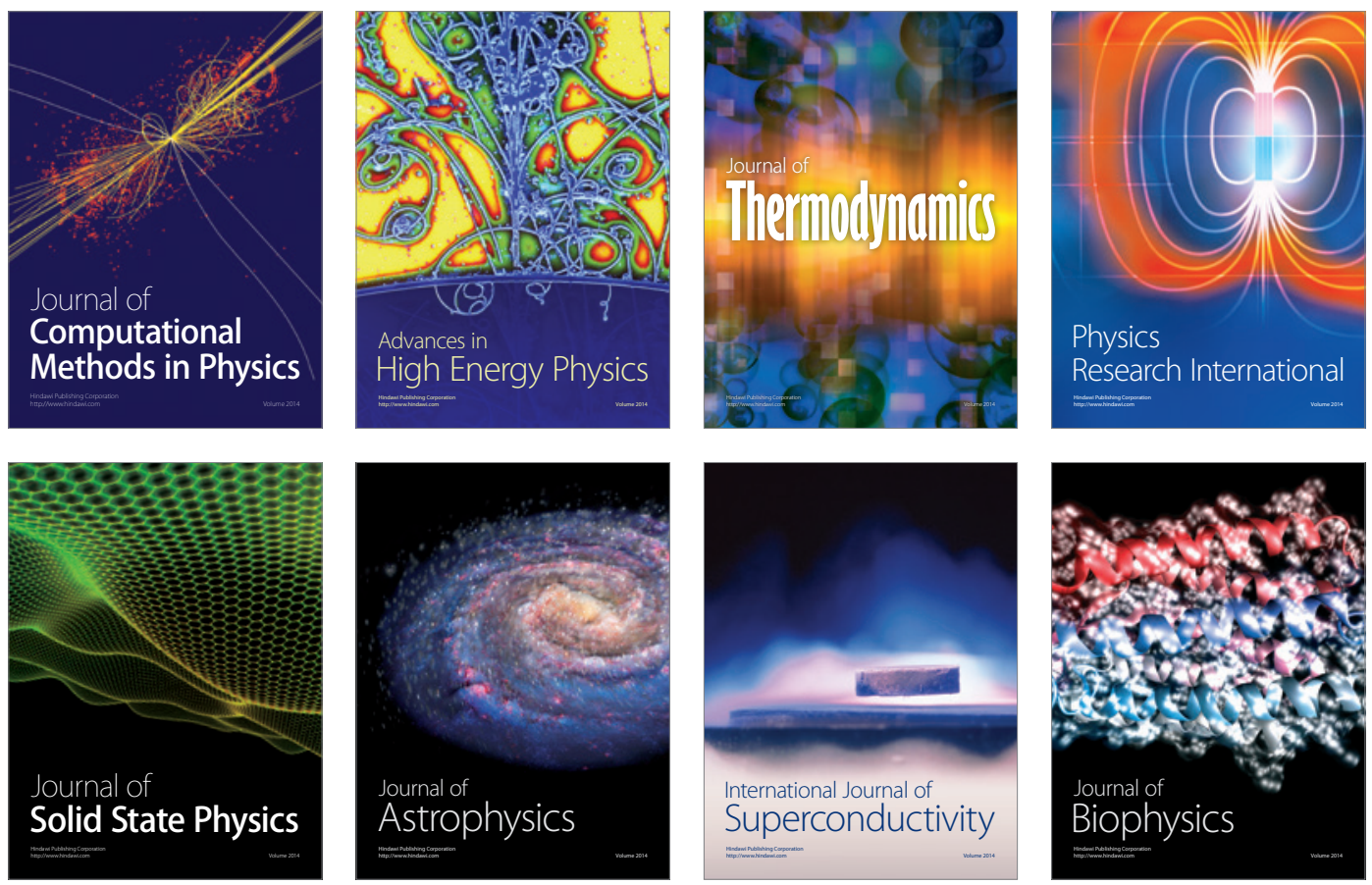
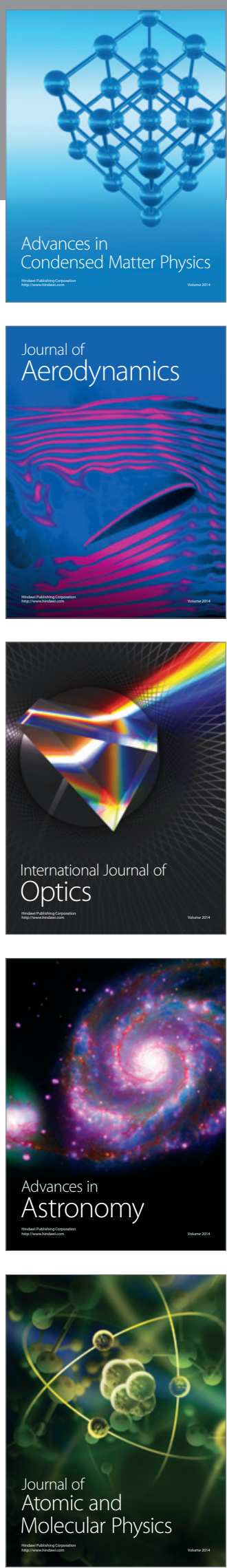